\title{
Highly Diastereoselective Oxidative Addition of Methyl Iodide to a Chiral Square Planar Complex
}

\author{
Mesfin Janka, Abdurrahman Ç. Atesin, Daniel J. Fox, Christine Flaschenriem, William W. \\ Brennessel, and Richard Eisenberg* \\ Department of Chemistry, University of Rochester, Rochester, New York 14627
}

\section{Supporting Information}

General Procedures and Materials. Chloroform- $d$, dichloromethane- $d_{2}$, DMSO- $d_{6}$, and toluene- $d_{8}$ were purchased from Cambridge Isotope Laboratories. Methyl iodide was purchased from Aldrich Chemical Co. (2S, 3S)-(-)-bis(diphenylphosphino)butane ((S,S)chiraphos), (-)-1,2-bis((2S,5S)-2,5-di-i-propylphospholano)benzene $((S, S)$-i-Pr-duphos), and (+)-1,2-bis $((2 R, 5 R)$-2,5-di-i-propylphospholano)benzene $((R, R)$-i-Pr-duphos $)$ were obtained from Strem Chemical Company and used as received. Unless otherwise stated, all reactions and manipulations were performed in dry glassware under a nitrogen atmosphere using either standard Schlenk techniques or an inert-atmosphere glovebox. THF, $\mathrm{CH}_{2} \mathrm{Cl}_{2}$, diethyl ether, and hexanes were purified as described by Grubbs. ${ }^{1}$ All NMR spectra were recorded on Avance 400 or $500 \mathrm{MHz}$ spectrometers. ${ }^{1} \mathrm{H}$ chemical shifts (in ppm) are relative to tetramethylsilane and referenced using chemical shifts of residual solvent resonances. ${ }^{31} \mathrm{P}$ chemical shifts (in ppm) are relative to an external $85 \%$ solution of phosphoric acid in the appropriate solvent.

Synthesis of IrI(CO)(chiraphos) (4). A modification of the published procedure ${ }^{2}$ was followed. The reaction was conducted under an atmosphere of CO. A $250 \mathrm{~mL}$ three-neck flask containing a stir-bar was fitted with a $125 \mathrm{~mL}$ pressure equalizing addition funnel and a gasinlet. [TBA] $\left[\operatorname{Ir}(\mathrm{CO})_{2} \mathrm{I}_{2}\right](1.00 \mathrm{~g}, 1.34 \mathrm{mmol})$ was added as a solid to the 3 -neck flask and chiraphos (0.57 $\mathrm{g}, 1.34 \mathrm{mmol})$ was added to the addition funnel. The iridium complex was dissolved in $50 \mathrm{~mL}$ of $\mathrm{THF}$, cooled to $-78^{\circ} \mathrm{C}$ and purged for $5 \mathrm{~min}$ with $\mathrm{CO}$. The chiraphos ligand was dissolved in $30 \mathrm{~mL}$ of THF and added dropwise over a period of $1 \mathrm{~h}$. After $1 \mathrm{~h}$ of stirring at $-78^{\circ} \mathrm{C}$, the dry ice bath was removed and the reaction mixture was allowed to warm to room temperature for one more hour. It was then concentrated in vacuo to $10 \mathrm{~mL}$ and $\mathrm{N}_{2^{-}}$ degassed ethanol $(50 \mathrm{~mL})$ was added. The solvent was concentrated in vacuo to about $15 \mathrm{~mL}$, and the flask was cooled to $-35^{\circ} \mathrm{C}$ in the freezer. The orange solid was isolated on a frit in air 
and washed with cold ethanol, hexanes and then cold diethyl ether. Yield: $0.83 \mathrm{~g}(81 \%)$. ${ }^{1} \mathrm{H}$ NMR $\left(\mathrm{CD}_{2} \mathrm{Cl}_{2}\right)$ 7.96-7.34 (m, 20H, phenyl), 2.39-2.36 (m, 1H, $\left.\mathrm{PCHCH} \mathrm{CHCH}_{3} \mathrm{P}\right), 2.16-2.11$ $\left(\mathrm{m}, 1 \mathrm{H}, \mathrm{PCHCH}_{3} \mathrm{CHCH}_{3} \mathrm{P}\right), 1.08-0.96\left(\mathrm{~m}, 6 \mathrm{H}, \mathrm{PCHCH}_{3} \mathrm{CHCH}_{3} \mathrm{P}\right) ;{ }^{11} \mathrm{P}\left\{{ }^{1} \mathrm{H}\right\} \mathrm{NMR}\left(\mathrm{CD}_{2} \mathrm{Cl}_{2}\right)$ $48.2\left(\mathrm{~d},{ }^{2} J_{\mathrm{P}-\mathrm{P}}=25.0 \mathrm{~Hz}\right), 44.5\left(\mathrm{~d},{ }^{2} J_{\mathrm{P}-\mathrm{P}}=25.0 \mathrm{~Hz}\right)$; IR (KBr pellet): $1971 \mathrm{~cm}^{-1}$.

Synthesis of $\operatorname{IrI}(\mathbf{C O})($ duphos) (5). The same procedure was followed as described above for the synthesis of 4 with the following amounts: [TBA] $\left.\operatorname{Ir}(\mathrm{CO})_{2} \mathrm{I}_{2}\right](0.18 \mathrm{~g}, 0.24 \mathrm{mmol})$ in $10 \mathrm{~mL} \mathrm{THF}$, and duphos $(0.10 \mathrm{~g}, 0.24 \mathrm{mmol})$. Purified yield: $0.13 \mathrm{~g}(72 \%)$. ${ }^{1} \mathrm{H} \mathrm{NMR}$ $\left(\mathrm{CD}_{2} \mathrm{Cl}_{2}\right) 7.76\left(\mathrm{~d}, J_{\mathrm{H}-\mathrm{H}}=6.8 \mathrm{~Hz}, 2 \mathrm{H}\right.$, aromatic protons $), 7.51\left(\mathrm{~d}, J_{\mathrm{H}-\mathrm{H}}=6.8 \mathrm{~Hz}, 2 \mathrm{H}\right.$, aromatic protons), $3.26(\mathrm{~m}, 1 \mathrm{H}$, aliphatic proton), 2.60-2.00 (m, 10H, aliphatic proton) $1.83-1.63$ (m, $4 \mathrm{H}$, aliphatic proton), $1.49-1.45(\mathrm{~m}, 1 \mathrm{H}$, aliphatic proton), 1.10-.0.86 (m, 12H, aliphatic protons) $0.729\left(\mathrm{~d}, J_{\mathrm{H}-\mathrm{H}}=6.0 \mathrm{~Hz}, 12 \mathrm{H}\right) ;{ }^{31} \mathrm{P}\left\{{ }^{1} \mathrm{H}\right\} \mathrm{NMR}\left(\mathrm{CD}_{2} \mathrm{Cl}_{2}\right) 61.1\left(\mathrm{~d},{ }^{2} J_{\mathrm{P}-\mathrm{P}}=14.4 \mathrm{~Hz}\right), 59.1(\mathrm{~d}$, ${ }^{2} J_{\mathrm{P}-\mathrm{P}}=14.4 \mathrm{~Hz}$ ); IR (KBr pellet): $1967 \mathrm{~cm}^{-1}$. Anal. Calcd for $\mathrm{C}_{27} \mathrm{H}_{44} \mathrm{IIOP}_{2}: \mathrm{C}, 42.35 ; \mathrm{H}, 5.79$. Found C, 42.40; H, 5.81.

Synthesis of $\operatorname{Ir}\left(\mathrm{CH}_{3}\right) \mathbf{I}_{2}(\mathbf{C O})($ chiraphos) (6a and 6b). To a $100 \mathrm{~mL}$ round bottom flask containing a stir-bar, $\operatorname{IrI}(\mathrm{CO})$ (chiraphos) $(0.75 \mathrm{~g}, 0.97 \mathrm{mmol})$ and $30 \mathrm{~mL}$ of $\mathrm{CH}_{2} \mathrm{Cl}_{2}$ was added 10 equivalents of methyl iodide $(0.60 \mathrm{~mL}, 9.7 \mathrm{moml})$. The color immediately lightened from dark orange to yellow. The reaction mixture was stirred for $1 \mathrm{~h}$ at room temperature. It was concentrated in vacuo to about $10 \mathrm{~mL}$ and $20 \mathrm{~mL}$ of hexanes was then added. Complexes 6a and $\mathbf{6 b}$ were isolated as a yellow solid in a 1:1 ratio in $92 \%$ combined yield. Spectroscopic data for 6a: ${ }^{1} \mathrm{H} \mathrm{NMR}\left(\mathrm{CD}_{2} \mathrm{Cl}_{2}\right)$ 8.28-6.96 (m, $20 \mathrm{H}$, phenyl), $3.93\left(\mathrm{~m}, 1 \mathrm{H}, \mathrm{PCHCH}_{3} \mathrm{CHCH}_{3} \mathrm{P}\right)$, $2.62\left(\mathrm{~m}, 1 \mathrm{H}, \mathrm{PCHCH}_{3} \mathrm{CHCH}_{3} \mathrm{P}\right), 1.35-1.19$ (m, 3H, $\left.\mathrm{PCHCH}_{3} \mathrm{CHCH}_{3} \mathrm{P}\right), 1.04-0.99(\mathrm{~m}, 3 \mathrm{H}$, $\left.\mathrm{PCHCH}_{3} \mathrm{CHCH}_{3} \mathrm{P}\right), 0.81\left(\mathrm{t}, J_{\mathrm{P}-\mathrm{P}}=4.4 \mathrm{~Hz}, 3 \mathrm{H}, \mathrm{CH}_{3}\right) ;{ }^{31} \mathrm{P}\left\{{ }^{1} \mathrm{H}\right\} \mathrm{NMR}\left(\mathrm{CD}_{2} \mathrm{Cl}_{2}\right) 20.9\left(\mathrm{~d},{ }^{2} J_{\mathrm{P}-\mathrm{P}}=\right.$ $13.4 \mathrm{~Hz}), 3.4\left(\mathrm{~d},{ }^{2} J_{\mathrm{P}-\mathrm{P}}=13.4 \mathrm{~Hz}\right)$; IR (KBr pellet): $2056 \mathrm{~cm}^{-1}$. Spectroscopic data for $\mathbf{6 b}:{ }^{1} \mathrm{H}$ NMR $\left(\mathrm{CD}_{2} \mathrm{Cl}_{2}\right)$ 8.28-6.96 (m, $20 \mathrm{H}$, phenyl), $3.10\left(\mathrm{~m}, 2 \mathrm{H}, \mathrm{PCHCH}_{3} \mathrm{CHCH}_{3} \mathrm{P}\right), 1.35-1.19(\mathrm{~m}$, $\left.3 \mathrm{H}, \mathrm{PCHCH}_{3} \mathrm{CHCH}_{3} \mathrm{P}\right), 1.04-0.99\left(\mathrm{~m}, 3 \mathrm{H}, \mathrm{PCHCH}_{3} \mathrm{CHCH}_{3} \mathrm{P}\right), 0.81\left(\mathrm{t}, J_{\mathrm{P}-\mathrm{P}}=4.4 \mathrm{~Hz}, 3 \mathrm{H}\right.$, $\left.\mathrm{CH}_{3}\right) ;{ }^{31} \mathrm{P}\left\{{ }^{1} \mathrm{H}\right\} \mathrm{NMR}\left(\mathrm{CD}_{2} \mathrm{Cl}_{2}\right) 21.9\left(\mathrm{~d},{ }^{2} J_{\mathrm{P}-\mathrm{P}}=13.4 \mathrm{~Hz}\right), 4.1\left(\mathrm{~d},{ }^{2} J_{\mathrm{P}-\mathrm{P}}=13.4 \mathrm{~Hz}\right) ; \mathrm{IR}(\mathrm{KBr}$ pellet): $2056 \mathrm{~cm}^{-1}$.

Synthesis of $\operatorname{Ir}\left(\mathrm{CH}_{3}\right) \mathbf{I}_{2}(\mathbf{C O})($ duphos $)$ (7). To a $100 \mathrm{~mL}$ round bottom flask containing a stir-bar, $\operatorname{IrI}(\mathrm{CO})($ duphos $)(0.25 \mathrm{~g}, 0.33 \mathrm{mmol})$ and $15 \mathrm{~mL}$ of $\mathrm{CH}_{2} \mathrm{Cl}_{2}$ was added 10 equivalents of methyl iodide $(0.20 \mathrm{~mL}, 3.3 \mathrm{mmol})$. The reaction mixture was stirred for $10 \mathrm{~h}$ at room temperature. It was concentrated in vacuo to about $3 \mathrm{~mL}$, and $10 \mathrm{~mL}$ of hexanes was then added. Complexes $7 \mathbf{a}$ and $\mathbf{7 b}$ were isolated as a yellow solid in a 95:5 ratio in 83\% combined yield. Spectroscopic data for the major isomer of 7: ${ }^{1} \mathrm{H} \mathrm{NMR}\left(\mathrm{CD}_{2} \mathrm{Cl}_{2}\right) 7.94\left(\mathrm{t}, J_{\mathrm{H}-\mathrm{H}}=7.2 \mathrm{~Hz}\right.$, $1 \mathrm{H}$, aromatic proton), $7.76\left(\mathrm{t}, J_{\mathrm{H}-\mathrm{H}}=7.2 \mathrm{~Hz}, 1 \mathrm{H}\right.$, aromatic proton $), 7.56(\mathrm{~m}, 2 \mathrm{H}$, aromatic 
protons), $3.90(\mathrm{~m}, 2 \mathrm{H}$, aliphatic proton), $3.26(\mathrm{~m}, 2 \mathrm{H}$, aliphatic proton), 2.85-2.73 (m, $2 \mathrm{H}$, aliphatic protons), 2.56-1.9 (m, $8 \mathrm{H}$, aliphatic protons), 1.75 (m, $2 \mathrm{H}$, aliphatic proton), 1.30-0.88 $\left(\mathrm{m}, 12 \mathrm{H}\right.$, aliphatic protons), $0.74\left(\mathrm{t}, J_{\mathrm{P}-\mathrm{H}}=5.6 \mathrm{~Hz}, 3 \mathrm{H}, \mathrm{CH}_{3}\right), 0.67-0.55(\mathrm{~m}, 12 \mathrm{H}$, aliphatic protons); ${ }^{31} \mathrm{P}\left\{{ }^{1} \mathrm{H}\right\}$ NMR $\left(\mathrm{CD}_{2} \mathrm{Cl}_{2}\right) 43.5\left(\mathrm{~d},{ }^{2} J_{\mathrm{P}-\mathrm{P}}=8.1 \mathrm{~Hz}\right), 18.1\left(\mathrm{~d},{ }^{2} J_{\mathrm{P}-\mathrm{P}}=8.1 \mathrm{~Hz}\right)$. Spectroscopic data for the minor isomer of 7: ${ }^{31} \mathrm{P}\left\{{ }^{1} \mathrm{H}\right\}$ NMR $\left(\mathrm{CD}_{2} \mathrm{Cl}_{2}\right) 45.1\left(\mathrm{~d},{ }^{2} J_{\mathrm{P}-\mathrm{P}}=8.1\right.$ $\mathrm{Hz}), 20.2\left(\mathrm{~d},{ }^{2} J_{\mathrm{P}-\mathrm{P}}=8.1 \mathrm{~Hz}\right.$ ). IR (KBr pellet): $2037 \mathrm{~cm}^{-1}$. Anal. Calcd for $\mathrm{C}_{28} \mathrm{H}_{47} \mathrm{I}_{2} \mathrm{IrOP}_{2}: \mathrm{C}$, 37.05; H, 5.22. Found C, 37.10; H, 5.25.

Synthesis of $\left[\operatorname{Ir}(\mathbf{C O})_{2}(\right.$ duphos $\left.)\right]\left[S_{6 F}\right](8)$. The reaction was conducted under an atmosphere of CO. A $100 \mathrm{~mL}$ three-neck flask containing a stir-bar was fitted with a gas-inlet. $\operatorname{IrI}(\mathrm{CO})$ (duphos) (0.0300 g, $0.0392 \mathrm{mmol})$ was added as a solid to the 3-neck flask, dissolved in $20 \mathrm{~mL}$ of methylene chloride, and the solution was purged for $10 \mathrm{~min}$ with $\mathrm{CO}$. Upon the addition of solid $\mathrm{AgSbF}_{6}(0.0135 \mathrm{~g}, 0.0392 \mathrm{mmol}), \mathrm{AgI}$ precipitated. After removal of AgI by filtration, the product was obtained from the reaction solution as a pale orange solid upon the addition of diethyl ether. Yield: $0.033 \mathrm{~g}(95 \%)$. ${ }^{1} \mathrm{H}$ NMR $\left(\mathrm{CD}_{2} \mathrm{Cl}_{2}\right)$ 8.03-7.95 (m, 2H, aromatic protons), 7.95-7.88 (m, 2H, aromatic protons), 3.05-2.88 (m, 2H, aliphatic protons), 2.79-2.48 (m, 6H, aliphatic protons), 2.18-1.96 (m, 2H, aliphatic protons), 1.96-1.74 (m, 2H, aliphatic protons), 1.74-1.54 (m, 2H, aliphatic protons), 1.21 (d, $J_{\mathrm{H}-\mathrm{H}}=6.8 \mathrm{~Hz}, 6 \mathrm{H}$, aliphatic protons), $1.01\left(\mathrm{~d}, J_{\mathrm{H}-\mathrm{H}}=6.8 \mathrm{~Hz}, 6 \mathrm{H}\right.$, aliphatic protons $), 0.90\left(\mathrm{~d}, J_{\mathrm{H}-\mathrm{H}}=6.8 \mathrm{~Hz}, 6 \mathrm{H}\right.$, aliphatic protons), $0.69\left(\mathrm{~d}, J_{\mathrm{H}-\mathrm{H}}=6.8 \mathrm{~Hz}, 6 \mathrm{H}\right.$, aliphatic protons $) ;{ }^{31} \mathrm{P}\left\{{ }^{1} \mathrm{H}\right\} \mathrm{NMR}\left(\mathrm{CD}_{2} \mathrm{Cl}_{2}\right) 64.7(\mathrm{~s}) ; \mathrm{IR}\left(\mathrm{KBr}, \mathrm{cm}^{-}\right.$ 1) 2077 (s, CO), 2025 (s, CO). Anal. Calcd for $\mathrm{C}_{28} \mathrm{H}_{44} \mathrm{~F}_{6} \mathrm{IrO}_{2} \mathrm{P}_{2} \mathrm{Sb}$ : C, 37.26; H, 4.91. Found C, 37.35; H, 4.67.

General procedure for X-ray structural determinations. For each structure determination, the crystal was mounted under paratone-8277 oil on a glass fiber and immediately placed in a cold nitrogen stream at $100 \mathrm{~K}$ on the X-ray diffractometer. The X-ray intensity data were collected on a standard Bruker APEX II CCD area detector system equipped with a fine focus molybdenum-target X-ray tube operated at $1.5 \mathrm{~kW}(50 \mathrm{kV}, 30 \mathrm{~mA})$. Data were collected using a narrow frame method. The total data collection time was approximately $8 \mathrm{~h}$. Frames were integrated to a maximum $2 \theta$ angle with the Siemens SAINT program and corrected for absorption with the program SADABS. Each structure was solved by direct methods and refined employing full-matrix least-squares on $F^{2}$. All non-H atoms in each structure were refined with anisotropic thermal parameters. The hydrogen atoms were included in idealized positions.

Complex 5. The iodide and carbonyl ligands are modeled as disordered with each other (76:24) and one arm of the duphos ligand is modeled as disordered over two positions (53:47). 
Thermal constraints were imposed on both disorders.

(b)

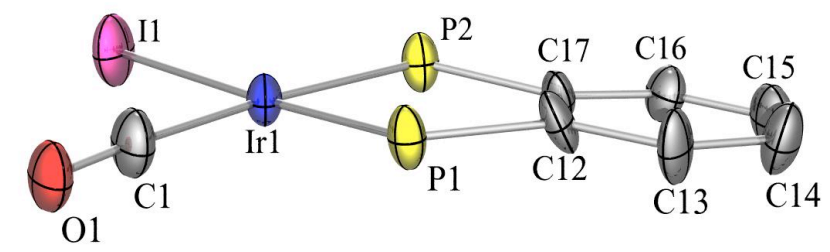

(a)

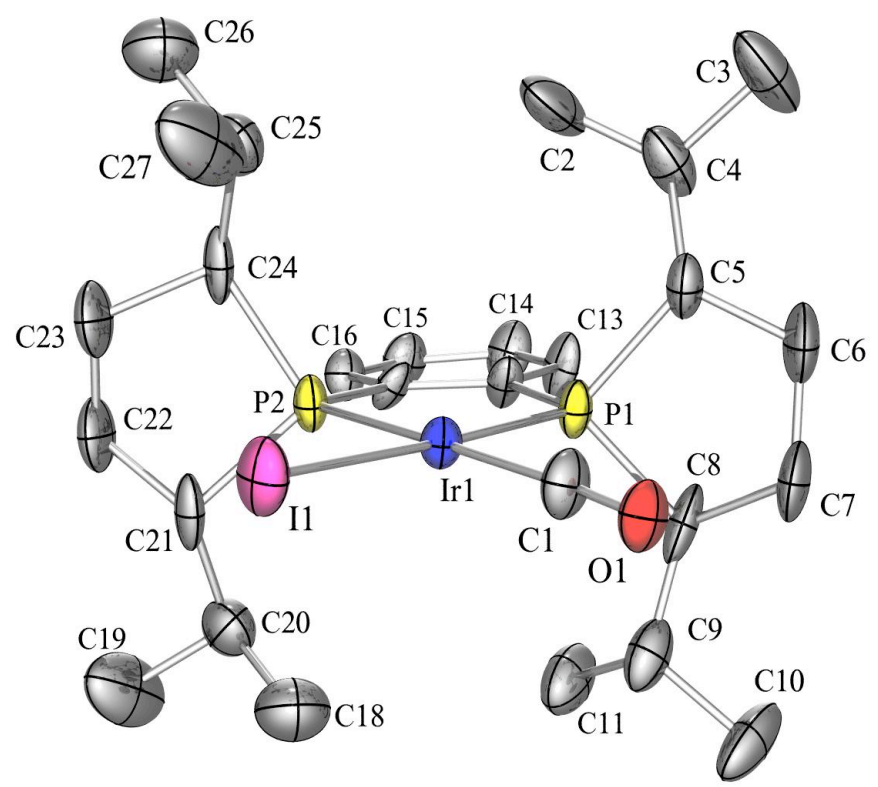

Figure S-1. (a) An ORTEP32/POVRAY representation of 5 showing $50 \%$ probability ellipsoids. For clarity, $\mathrm{H}$ atoms are not shown. (b) Selected part of the molecule. 
Table S-1. Crystal data and structure refinement for 5 .

Empirical formula

Formula weight

Temperature

Wavelength

Crystal system

Space group

Unit cell dimensions

Volume

Z

Density (calculated)

Absorption coefficient

$F(000)$

Crystal color, morphology

Crystal size

Theta range for data collection

Index ranges

Reflections collected

Independent reflections

Observed reflections

Completeness to theta $=30.51^{\circ}$

Absorption correction

Max. and min. transmission

Refinement method

Data / restraints / parameters

Goodness-of-fit on $F^{2}$

Final $R$ indices [I>2sigma $(I)]$

$R$ indices (all data)

Absolute structure parameter

Largest diff. peak and hole
$\mathrm{C}_{27} \mathrm{H}_{44} \mathrm{IIOP}_{2}$

765.66

100.0(1) K

$0.71073 \AA$

Orthorhombic

$P 2{ }_{1} 2_{1} 2_{1}$

$a=10.978(2) \AA \quad \alpha=90^{\circ}$

$b=14.296(3) \AA \quad \beta=90^{\circ}$

$c=18.263(4) \AA \quad \gamma=90^{\circ}$

2866.2(10) $\AA^{3}$

4

$1.774 \mathrm{Mg} / \mathrm{m}^{3}$

$5.864 \mathrm{~mm}^{-1}$

1496

orange, needle

$0.44 \times 0.12 \times 0.04 \mathrm{~mm}^{3}$

1.81 to $30.51^{\circ}$

$-15 \leq h \leq 15,-20 \leq k \leq 19,-25 \leq l \leq 25$

44717

$8612[R(\mathrm{int})=0.0606]$

6292

$99.4 \%$

Multi-scan

0.7993 and 0.1823

Full-matrix least-squares on $F^{2}$

8612 / 29 / 306

1.026

$R 1=0.0348, w R 2=0.0644$

$R 1=0.0635, w R 2=0.0710$

0.007(9)

1.195 and $-1.003 \mathrm{e} . \AA^{-3}$ 
Table S-2. Crystal data and structure refinement for the major isomer of 7.

Empirical formula

Formula weight

Temperature

Wavelength

Crystal system, space group

Unit cell dimensions

Volume

Z, Calculated density

Absorption coefficient

$\mathrm{F}(000)$

Crystal size

Theta range for data collection

Limiting indices

Reflections collected/unique

Completeness to theta $=28.30$

Absorption correction

Max. and min. transmission

Refinement method

Data / restraints / parameters

Goodness-of-fit on $\mathrm{F}^{2}$

Final $\mathrm{R}$ indices [I $>2 \operatorname{sigma}(\mathrm{I})]$

$\mathrm{R}$ indices (all data)

Absolute structure parameter

Largest diff. peak and hole

\section{$\mathrm{C}_{28} \mathrm{H}_{47} \mathrm{I}_{2} \mathrm{IrOP}_{2}$}

907.60

100(2) K

$0.71073 \mathrm{~A}$

Orthorhombic, P2(1)2(1)2(1)

$\mathrm{a}=10.6355(4) \AA \quad$ alpha $=90 \mathrm{deg}$.

$\mathrm{b}=11.1126(4) \AA \quad$ beta $=90 \mathrm{deg}$.

$\mathrm{c}=26.7012(9) \AA$ gamma $=90 \mathrm{deg}$.

3155.8(2) $\AA^{3}$

$4,1.910 \mathrm{mg} / \mathrm{m}^{3}$

$6.308 \mathrm{~mm}^{-1}$

1744

$0.38 \times 0.15 \times 0.13 \mathrm{~mm}$

1.53 to $28.30 \mathrm{deg}$.

$-14 \leq \mathrm{h} \leq 14,-14 \leq \mathrm{k} \leq 14,-35 \leq 1 \leq 35$

$42433 / 7752[\mathrm{R}(\mathrm{int})=0.0257]$

$99.9 \%$

Empirical

0.440 and 0.334

Full-matrix least-squares on $\mathrm{F}^{2}$

$7752 / 0 / 320$

0.881

$\mathrm{R} 1=0.0137, \mathrm{wR} 2=0.0308$

$\mathrm{R} 1=0.0146, \mathrm{wR} 2=0.0310$

$0.010(2)$

1.146 and $-0.403 \AA^{-3}$ 
(b)

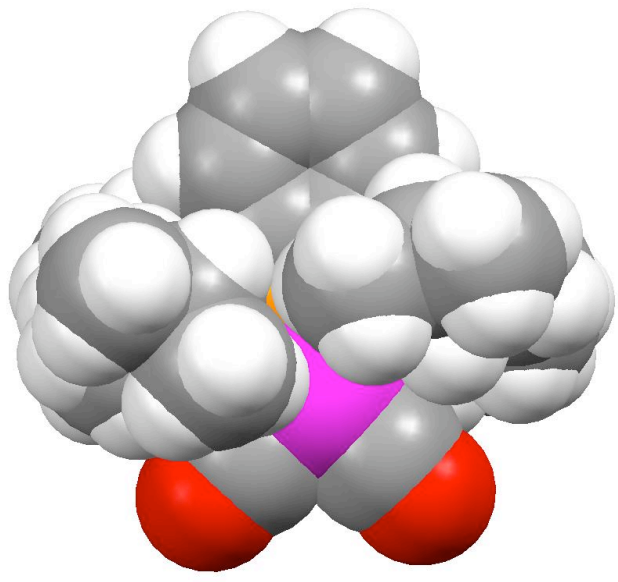

(a)

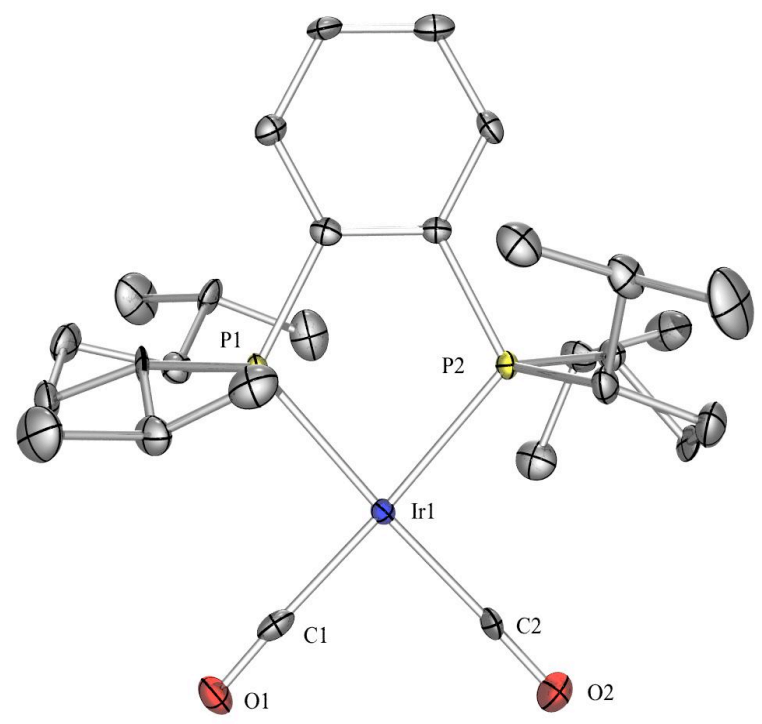

Figure S-2. . (a) An ORTEP32/POVRAY representation of 8 showing 50\% probability ellipsoids. For clarity, $\mathrm{H}$ atoms are not shown. (b) Space filling model of the molecule. 
Table S-3. Crystal data and structure refinement for $\mathbf{8}$.

Empirical formula

Formula weight

Temperature

Wavelength

Crystal system

Space group

Unit cell dimensions

Volume

Z

Density (calculated)

Absorption coefficient

$F(000)$

Crystal color, morphology

Crystal size

Theta range for data collection

Index ranges

Reflections collected

Independent reflections

Observed reflections

Completeness to theta $=30.51^{\circ}$

Absorption correction

Max. and min. transmission

Refinement method

Data / restraints / parameters

Goodness-of-fit on $F^{2}$

Final $R$ indices $[I>2 \operatorname{sigma}(I)]$

$R$ indices (all data)

Absolute structure parameter

Largest diff. peak and hole

\section{C28 H44 F6 Ir O2 P2 Sb}

902.52

100.0(1) K

$0.71073 \AA$

Orthorhombic

$P 2{ }_{1} 2_{1} 2_{1}$

$a=8.7159(18) \AA \quad \alpha=90^{\circ}$

$b=11.833(2) \AA \quad \beta=90^{\circ}$

$c=31.627(7) \AA \quad \gamma=90^{\circ}$

3262.0(12) $\AA^{3}$

4

$1.838 \mathrm{Mg} / \mathrm{m}^{3}$

$5.060 \mathrm{~mm}^{-1}$

1760

yellow-orange, block

$0.40 \times 0.34 \times 0.18 \mathrm{~mm}^{3}$

1.29 to $30.51^{\circ}$

$-12 \leq h \leq 12,-16 \leq k \leq 16,-45 \leq l \leq 43$

49306

$9785[R($ int $)=0.0552]$

9355

$99.5 \%$

Multi-scan

0.4028 and 0.1468

Full-matrix least-squares on $F^{2}$

9785 / 0 / 369

1.082

$R 1=0.0342, w R 2=0.0805$

$R 1=0.0361, w R 2=0.0816$

$0.009(6)$

1.468 and $-2.083 \mathrm{e} . \AA^{-3}$ 


\section{References:}

(1) Pangborn, A. B., Giardello, M. A., Grubbs, R. H., Rosen, R. K., Timmers, F. J. Organometallics 1996, 15, 1518-1520.

(2) Kunin, A. J., Farid, R., Johnson, C. E., Eisenberg, R. J. Am. Chem. Soc. 1985, 107, 5315- 5317. 